\title{
ESTRATEGIAS CREATIVAS EN PSICOTERAPIA BREVE
}

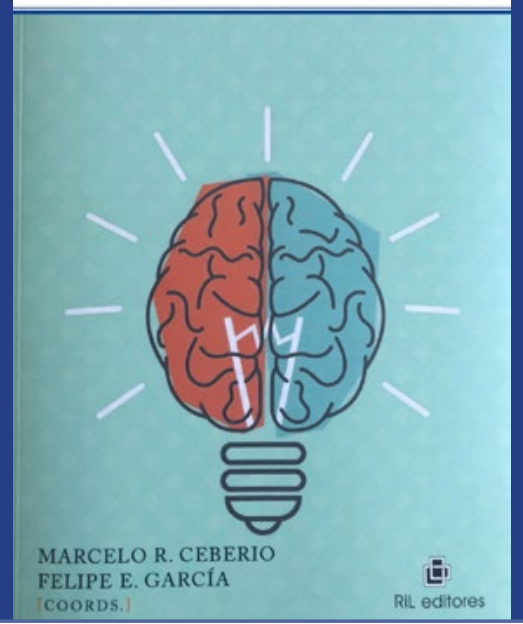

Editores:

Marcelo R. Ceberio ${ }^{1}$ y Felipe E. García ${ }^{2}$

Editorial: RIL Editores Chile. 2018

Número de páginas: 426

ISBN: 978-956-01-0523-3

por Pablo Concha Ponce ${ }^{3}$

La presente recensión está dedicada al último libro de los destacados terapeutas latinoamericanos Marcelo R. Ceberio y Felipe E. García, quienes desde Argentina y Chile, respectivamente, se han encargado de reunir las voces de entusiastas psicoterapeutas de América Latina que presentan diversas maniobras terapéuticas basadas en los conceptos tradicionales de la terapia sistémica estratégica y terapia breve, adaptadas a las problemáticas que emergen en nuestro contexto latinoamericano.

En mi experiencia como docente de Intervención Clínica y Terapia Sistémica Breve (TSB) en pregrado de psicología y en la formación de postítulos y diplomados para psicólogos y trabajadores sociales titulados, resulta necesario tener material bibliográfico actualizado y que condense de forma sintética y ordenada

\footnotetext{
${ }^{1}$ Doctor en Psicología de la Universidad de Barcelona y de la Universidad Kennedy. Master en Terapia Familiar (Universidad Autónoma de Barcelona) y Psicoinmunoendocrinología (Universidad Favaloro). Se entrenó en el modelo sistémico en el Mental Research Institute de Palo Alto (USA). Director Académico e Investigación de la Escuela Sistémica Argentina. Profesor en diversas universidades e institutos de Latinoamérica, Europa y Estados Unidos.

${ }^{2}$ Magister y Doctor en Psicología de la Universidad de Concepción. Postítulos en terapia estratégica breve e hipnosis clínica. Director del Centro de Estudios Sistémicos CESIST. Académico e investigador de la Universidad Santo Tomás sede Concepción, Chile. Docente de postgrados, postítulos y diplomados en Chile, Perú, Bolivia y Ecuador.

${ }^{3}$ Psicólogo, Licenciado en Psicología, Universidad Autónoma de Chile. Doctorando en Psicología, Universidad de Flores, Buenos Aires, Argentina. Cursando Master Psicoterapia Breve Estratégica, Centro Di Terapia Strategica de Arezzo, Italia. Supervisor en Psicoterapia Sistémica Constructivista, CAPSIS Chile \& Grupo Palo Alto, MRI USA. Postítulo en Psicoterapia Estratégica Breve, Instituto Milton Erickson de Santiago. Académico y Supervisor Clínico en la Escuela de Psicología de la Universidad Autónoma de Chile, Talca. Correspondencia dirigirla a: pablo.concha@uautonoma.cl
} 
las maniobras terapéuticas que se están trabajando. A pesar de que existe un número abundante de publicaciones sobre TSB en diversas partes del mundo, cuando observamos la realidad en Latinoamérica esta obra cobra importancia, pues se transforma en un trabajo que logra presentar elementos históricos, epistemológicos, teóricos y prácticos desde los distintos modelos que forman parte de la TSB, quedando finalmente bien desarrollados y sintetizados en un solo libro.

Este libro presenta diversas áreas de aplicación de la TSB, conformado por 22 capítulos, divididos en 5 partes, de autores de Argentina, Chile, México, Bolivia, Perú y Colombia. Como lo plantea Reynaldo Perrone -argentino que en la actualidad vive en Europa- en el prólogo, cada capítulo es una participación remarcable que contribuye a componer una obra original digna de pertenecer a la biblioteca personal de todo psicoterapeuta (veterano o debutante, profesor o estudiante, de orientación sistémica o psicodinámica) y, evidentemente, de los centros de enseñanza.

Cada capítulo está escrito por expertos en la materia, que presentan aplicaciones a contextos locales, problemáticas emergentes, incorporando áreas de trabajo con niños, niñas y adolescentes, adultos, familias y parejas, presentando de forma rigurosa los principios terapéuticos básicos, la práctica crítica y ética en el trabajo con las personas. Resulta importante destacar que es un libro útil no sólo para psicólogos clínicos, sino también psicólogos de otras áreas y también profesionales de salud mental en general.

En la primera parte, que lleva por nombre "Disquisiciones prácticas en terapia sistémica", encontramos capítulos que invitan a la reflexión respecto del rol del terapeuta en el proceso de cambio de las personas. El aporte "Errores, dificultades, problemas y síntomas" de Marcelo Ceberio, es un análisis teórico respecto a situaciones comunes en los procesos terapéuticos, la comunicación humana y distinciones en la conceptualización de problemas dentro del modelo estratégico, aportando maniobras en la búsqueda del cambio y resolución de problemas.

Aportando a la reflexión y análisis teórico, Daniel Wilhelm ofrece una actualización -tan necesaria para la base epistemológica de la TSB- de los aportes del famoso y experimentado Hipnoterapeuta Milton Erickson a la psicoterapia estratégica actual, exhibiendo pasajes históricos y su trascendencia a través de los modelos actuales de TSB.

Finalmente, Ruperto Charles entrega orientaciones prácticas y didácticas que permiten innovar en el desarrollo de cada sesión a través de la elaboración de encuadres y tareas en el trabajo terapéutico.

En la segunda parte, respecto a "Modelos creativos en terapia sistémica", Felipe Gutiérrez presenta la relevancia que tiene el uso del humor y potenciar la creatividad en el ejercicio terapéutico como estrategia viable en el proceso de 
búsqueda de soluciones, de la forma más rápida posible. A través de casos clínicos, el autor logra transmitir ideas innovadoras respecto a los recursos terapéuticos para establecer preguntas y conversaciones aparentemente "simples" pero que impactan fuertemente en la alianza terapéutica.

Los colegas Marina Gómez, Belén González y Miguel Ángel Haza presentan su proyecto "La imagen fotográfica como técnica de intervención psicoterapéutica", quienes a través del uso del retrato fotográfico nos permiten apreciar cómo la psicoterapia actual entra en tensión con los paradigmas de las escuelas clásicas de terapia sistémica, a través de nuevas formas de expresión como el uso de la imagen.

En el capítulo 6, Beatriz Labrit ofrece una provocadora integración de recursos cognitivos-sistémicos del Análisis Transaccional (AT) en la psicoterapia de grupo, ofreciendo nuevas herramientas que enriquecen la práctica sistémica en general. Finalmente, Margarita Dubourdieu presenta un capítulo motivante, fruto de su experiencia de trabajo desde la Psico-neuro-inmuno-endocrinología, en el cual aborda la importancia de la mirada sistémica en la comprensión de cuadros como el estrés, así como también fundamenta la integración en psicoterapia en respuesta a dicha situación.

En la tercera parte, sobre "Adicción a las drogas y problemáticas infantojuveniles", Rodrigo Mardones extiende una invitación a la reflexión respecto a la experiencia del duelo en la infancia, integrando aspectos contextuales en su intervención. Ofrece una mirada ecológica en su trabajo, respetuosa, sensible y crítica en su ejercicio terapéutico.

En el capítulo 9, la mexicana Marta Campillo expone su experiencia de intervención con jóvenes que se cortan la piel, a través de la técnica del "árbol de la vida", expresión gráfica en búsqueda de recursos personales que, además, permite abrir conversaciones de externalización del problema, visibilizar sus habilidades, destrezas, esperanzas y sueños.

En el capítulo 10, Oliver Peñafiel presenta un caso clínico desde la terapia narrativa en un adolescente con intento suicida. En este sentido, cabe resaltar la sensibilidad y el respeto que el autor expone en el diálogo con los adolescentes.

El capítulo 11 Felipe E. García ofrece un análisis de una problemática tan presente en nuestra sociedad, en donde confluyen dilemas familiares y estrés académico en adolescentes. Finalmente, entrega ideas de intervención basadas en la evidencia científica que permiten ayudar a los jóvenes y sus familias a enfrentar la ansiedad.

En el capítulo 12, Jorge Ayala ofrece un panorama práctico respecto a cómo construir una relación terapéutica con personas en visita involuntaria por consumo de drogas. Invita a evaluar de forma crítica conceptos como "resistencia 
al cambio", personas "desafiantes" y "desmotivadas", incorporando nuevas distinciones desde la terapia breve centrada en soluciones, poniendo énfasis en aquello que funciona y la persona como centro de la conversación, con su visión de mundo, experiencias y saberes de vida.

Finalmente, Ricardo Celis expone su capítulo "Miradas contemporáneas sobre la pauta adictiva", una conceptualización al margen de la tradición psicopatológica psiquiátrica incorporando elementos de la complejidad que existe en la pauta adictiva, desde las relaciones familiares hasta una mirada existencial de la persona con una dinámica adictiva.

En la cuarta parte, respecto al trabajo en "Duelo y trauma en adultos", nuevamente aparece un capítulo de Felipe E. García, en el que exhibe sus destrezas terapéuticas, conceptuales y relacionales en un caso clínico de una persona viviendo un duelo, siendo capaz de exponer de forma clara y ordenada las maniobras terapéuticas realizadas desde la intervención hipnótica y terapia estratégica.

A continuación, en el capítulo 15, Valeska Aponte presenta una propuesta de abordaje en duelo anticipado en cuidadores de pacientes con enfermedad de Alzheimer. El trabajo se presenta como una revisión teórica del duelo anticipado, para posteriormente realizar una propuesta de intervención con los cuidadores.

En el capítulo 16, los peruanos César Vásquez y Teresa Mendo presentan un caso clínico de experiencias vitales críticas, abordado principalmente desde la terapia estratégica breve. En el capítulo 17, Arnoldo Téllez, Teresa Sánchez y Leticia Jaime presentan los beneficios de la hipnoterapia en pacientes con cáncer de mama, poniendo énfasis en la aproximación biopsicosocial del tratamiento de la enfermedad para, así, mejorar su calidad de vida.

En el capítulo 18, el chileno Luis de la Barra ofrece una reflexión teóricapráctica del doble vínculo en mujeres con diagnóstico de trastorno límite de la personalidad, a través del análisis de experiencias en el trabajo con la terapia estratégica breve.

En la quinta y última parte del libro, en lo relativo a "Problemas de parejas", encontramos la conceptualización que el reconocido terapeuta Reynaldo Perrone realiza del deseo, placer y goce en las pautas relacionales en las parejas. El capítulo se presenta como una tentadora invitación hacia las actualizaciones teóricas sobre el erotismo y seducción, información presentada en un lenguaje claro y sencillo, que todo terapeuta de parejas quisiera conocer para incorporar en su trabajo.

En el capítulo 20, Felipe E. García, Angélica Guzmán y Walter Jara ofrecen una guía de intervención sistémica breve en la infidelidad y reconciliación. En el desarrollo de un caso clínico, demuestran el valioso trabajo realizado, presentando testimonios y relatos de los participantes en su proceso de cambio. 
En el capítulo 21, el reconocido terapeuta boliviano Bismarck Pinto, presenta una actualización teórica-práctica de la psicoterapia de pareja centrada en soluciones y emancipación femenina.

Luego, en el capítulo 22, Felipe Rodríguez y Nataly Torres presentan una propuesta de intervención narrativa en la búsqueda de una relación estable en la adultez joven.

Finalmente, en el capítulo 23, nuevamente Bismarck Pinto ofrece un texto provocador, ahora enfocado en el análisis de "Amores peligrosos", que aborda el concepto del amor y realizando distinciones en las personas que se encuentran inmersas en un lazo amoroso peligroso.

Como ya se mencionó anteriormente, estamos frente a un libro que logra la transmisión sólida, coherente y con una fuerte base teórica del trabajo realizado desde la TSB en Latinoamérica, dando respuesta a las problemáticas locales, visibilizando la reflexión y el aporte al conocimiento que desde esta parte del mundo se está llevando a cabo. Algunos capítulos se transforman en una guía de procedimientos prácticos que todo terapeuta novel o experimentado sabrá valorar, pues están escritos en un lenguaje amigable y sencillo, que permite al lector comprender cada propuesta e impregnarse de la experiencia de cada terapeuta en su noble esfuerzo por trasmitir sus conocimientos. 


\section{Como citar este artículo}

Concha, P. (2018). Estrategias creativas en psicoterapia breve. Revista Pensamiento y Acción Interdisciplinaria, 4(1), 121-125.

DOI: http://doi.org/10.29035/pai.4.1.121

\section{Dirección de correspondencia}

\section{Pablo Concha Ponce}

Psicólogo. Máster en Psicoterapia Breve Estratégica.

Académico y Supervisor Clínico en la Escuela de Psicología, Universidad

Autónoma de Chile, Talca.

Contacto:

pablo.concha@uautonoma.cl

Recibido: 31/07/2018

Aceptado: 25/09/2018 